\title{
Intégrer les données probantes à la pratique avec le Comité consultatif national sur les infections transmissibles sexuellement et par le sang
}

\author{
Shamila Shanmugasegaram ${ }^{1 *}$, Stephan Gadient ${ }^{1}$, Margaret Gale-Rowe ${ }^{1}$
}

\section{Résumé}

Depuis plus de 30 ans, le gouvernement du Canada élabore des lignes directrices sur les infections transmissibles sexuellement et par le sang (ITSS) en collaboration avec un groupe d'experts en la matière. Ce groupe d'experts fournit des conseils à l'Agence de la santé publique du Canada (l'Agence) de 2004 à 2019, et devient le Comité consultatif national sur les ITSS (CCN-ITSS) en 2019. Le CCN-ITSS appuie le mandat de prévention et de contrôle des maladies infectieuses de l'Agence en fournissant des conseils pour l'élaboration de lignes directrices sur les ITSS. La méthodologie pour l'élaboration des recommandations du CCN-ITSS évolue vers un processus plus rigoureux, systématique et transparent, qui est conforme aux normes actuelles d'élaboration de lignes directrices. Elle s'appuie également sur, et est harmonisée avec, les méthodes de plusieurs autres concepteurs de lignes directrices. La méthodologie utilise l'approche GRADE (Grading of Recommendations, Assessment, Development and Evaluation), s'il y a lieu, pour l'examen des données probantes et l'élaboration des recommandations. Les recommandations sont publiées sur le site Web canada.ca avec l'énoncé du CCN-ITSS précisant la méthodologie et les données probantes utilisées pour leur élaboration. Ce processus permet d'assurer que les recommandations fondées sur des données probantes, fournies par l'Agence aux professionnels de soins primaires et de santé publique, sont crédibles.
Cette oeuvre est mise à la disposition selon les termes de la licence internationale Creative Commons Attribution 4.0

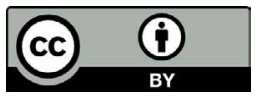

\section{Affiliation}

${ }^{1}$ Centre de la lutte contre les maladies transmissibles et les infections, Agence de la santé publique du Canada, Ottawa, ON

\author{
*Correspondance : \\ shamila.shanmugasegaram@ \\ canada.ca
}

Citation proposée : Shanmugasegaram S, Gadient S, Gale-Rowe M. Intégrer les données probantes à la pratique avec le Comité consultatif national sur les infections transmissibles sexuellement et par le sang. Relevé des maladies transmissibles au Canada 2020;46(11/12):449-53. https://doi.org/10.14745/ccdr.v46i1112a06f Mots-clés : lignes directrices fondées sur des données probantes, organisme consultatif externe, méthodologie pour les lignes directrices, prévention et contrôle des infections, infections transmissibles sexuellement et par le sang

\section{Introduction}

Les infections transmissibles sexuellement et par le sang (ITSS) demeurent une menace pour la santé publique des Canadiens. Les taux de chlamydia, de gonorrhée et de syphilis ont augmenté de façon constante au cours des dernières années (1), et plusieurs provinces et territoires ont déclaré des éclosions de syphilis en 2018 (2) et en 2019 (3). L'Agence de la santé publique du Canada (l'Agence) assure un leadership national en matière de prévention et de contrôle des ITSS en élaborant des lignes directrices de santé publique fondées sur des données probantes. Les recommandations émises dans ces lignes directrices sont élaborées, avec le soutien de l'Agence, par le Comité consultatif national sur les ITSS (CCN-ITSS). Le CCN-ITSS est un organisme consultatif externe composé d'experts en la matière provenant de partout au Canada. Cet article décrit le nouveau processus d'élaboration de recommandations sur les ITSS que I'Agence et le CCN-ITSS utilisent.

\section{Contexte}

Un groupe d'experts fournit des conseils au gouvernement du Canada depuis plus de 30 ans. Le premier comité consultatif, le Comité consultatif interdisciplinaire d'experts sur les maladies transmises sexuellement (MTS) chez les enfants et les jeunes, fut créé en 1986 par Santé et Bien-être social Canada afin de fournir des avis et des conseils sur la prévention et le contrôle des MTS. En 1988, ce comité a publié les premières Directives canadiennes pour le traitement des maladies transmises sexuellement chez les nouveau-nés, les enfants, les adolescents et les adultes (4). Bien que ce comité consultatif ait été dissous en 1991, le Groupe de travail d'experts sur les MTS a été mis sur pied en 1998 sous l'autorité du Laboratoire de lutte contre la maladie de Santé Canada dans le but d'élaborer des lignes directrices.

Suite à la création de l'Agence en 2004, le Groupe de travail d'experts sur les MTS a commencé à fournir des conseils à 
Cette nouvelle approche s'appuie sur les normes de pratiques

l'Agence, et le nom a été changé pour celui de Groupe de travail d'experts pour les Lignes directrices canadiennes sur les infections transmissibles sexuellement. En 2019, le Groupe de travail d'experts est devenu le CCN-ITSS, un organisme consultatif externe officiel établi en vertu des principes et des exigences prévus dans les lois et politiques du gouvernement fédéral (5).

\section{Mandat et composition}

Pour soutenir l'Agence dans son mandat de prévention et de contrôle les maladies infectieuses au Canada, le CCN-ITSS fournit, de manière continue et en temps opportun, des conseils et des recommandations pour l'élaboration de lignes directrices sur les ITSS. L'Agence conserve tous le pouvoir décisionnel et décide de la façon dont elle utilisera les recommandations et conseils de l'organisme consultatif externe.

Le CCN-ITSS s'acquitte de son mandat comme suit : effectue l'examen de la littérature épidémiologique et scientifique sur les ITSS ainsi que les données probantes sur les stratégies de prévention spécifiques, les diagnostics et les traitements; donne des conseils fondés sur les meilleures données probantes disponibles dans la littérature ou selon l'expertise spécialisée et l'expérience pratique des membres lorsqu'il y a un manque de données probantes; et conseille l'Agence sur les enjeux actuels et émergents relatifs aux ITSS.

Le CCN-ITSS est composé de 15 membres votants ayant une expertise dans les domaines de l'épidémiologie des soins de santé, des maladies infectieuses, de la microbiologie médicale, des diagnostics de laboratoire, de la pharmacologie, de l'obstétrique et de la gynécologie, de la pédiatrie, des soins primaires, de la psychologie et de la santé publique. Le recrutement est effectué pour s'assurer que les membres possèdent un éventail de connaissances, d'expertises, d'expériences, et de perspectives. La représentation géographique est également prise en compte lors du recrutement puisque que les enjeux peuvent varier d'une région à l'autre du Canada. L'Agence («le Secrétariat») évalue et gère les conflits d'intérêts des membres du CCN-ITSS qui doivent déclarer tout conflit d'intérêts au moment de se joindre et annuellement, et au début de chaque rencontre pour maintenir l'impartialité du comité.

\section{Processus d'élaboration de recommandations}

La méthodologie d'élaboration de recommandations du CCN-ITSS évolue vers un processus plus rigoureux, systématique et transparent pour formuler des recommandations crédibles. exemplaires en matière d'élaboration de lignes directrices (6-9) et sur la méthodologie de plusieurs autres concepteurs de lignes directrices (10-16). Un manuel décrivant la méthodologie à utiliser a été rédigé, dont le résumé est illustré à la figure 1.

Figure 1 : Processus d'élaboration de recommandations

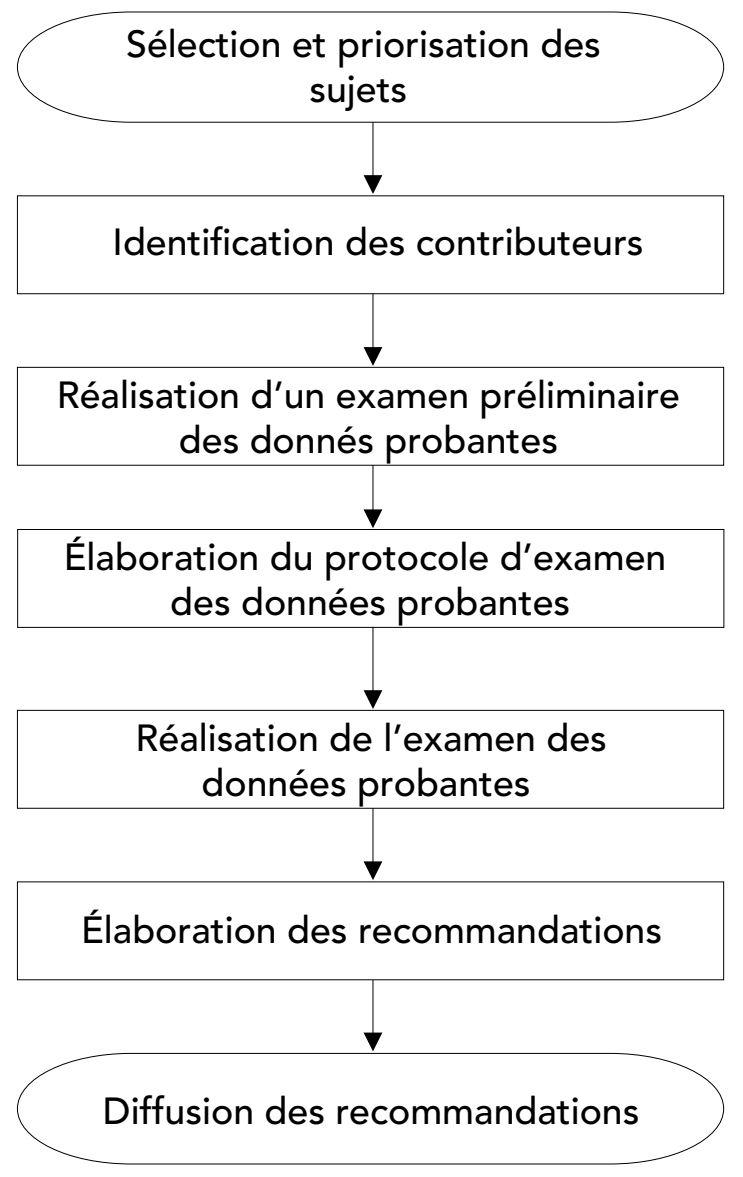

Chaque année (ou au besoin), le Secrétariat effectue un exercice de sélection et de priorisation des sujets afin de déterminer les recommandations existantes à réaffirmer ou mettre à jour, ou les nouvelles recommandations à élaborer au cours de la prochaine année financière. La figure 2 illustre l'exercice de sélection et de priorisation des sujets. Cet exercice comporte les étapes suivantes :

1. Solliciter des sujets pour l'élaboration de recommandations

2. Trier les sujets en fonction de la portée des lignes directrices sur les ITSS et des mandats et priorités de l'Agence

3. Compléter l'outil d'évaluation des sujets, qui comprend des questions sur la disponibilité des données probantes et de l'épidémiologie, pour aider le CCN-ITSS à classer les sujets par ordre de priorité

4. Classer (à répéter, au besoin) les sujets et discuter des résultats (CCN-ITSS) 
5. Déterminer (le Secrétariat) la liste finale des sujets pour l'élaboration de recommandations, obtenir l'approbation de l'Agence et partager cette liste avec le CCN-ITSS. Les sujets qui n'ont pas été jugés prioritaires pour l'élaboration de recommandations seront examinés de nouveau lors du prochain exercice de priorisation des sujets, avec toute nouvelle suggestion

\section{Figure 2 : Exercice de sélection et de priorisation}

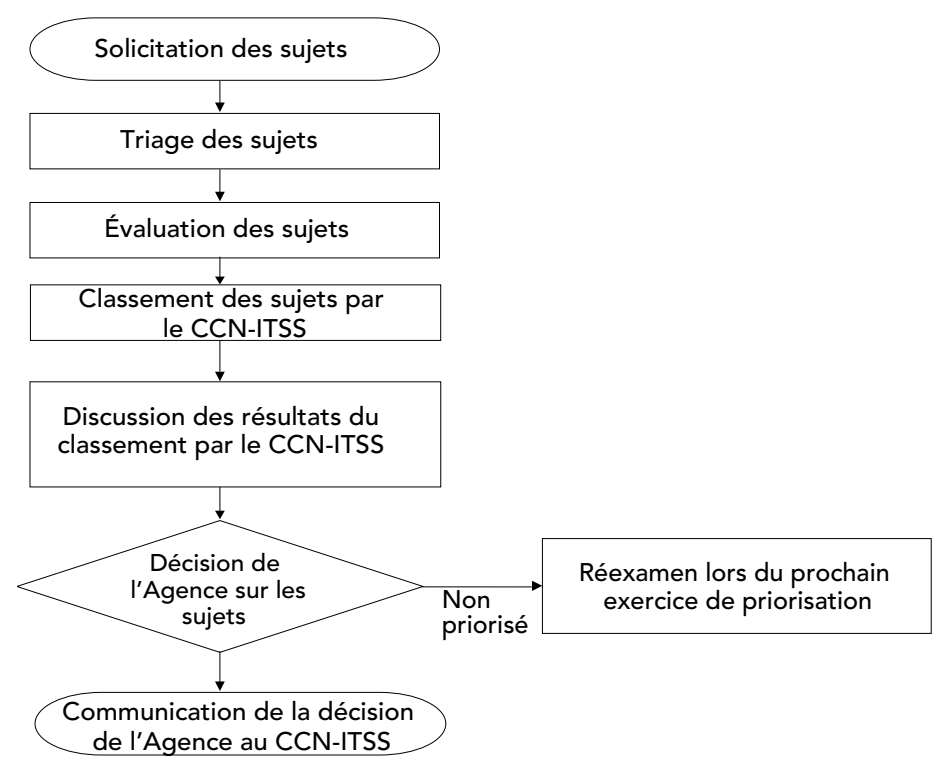

Abréviations : I'Agence, I'Agence de la santé publique du Canada; CCN-ITSS, Comité consultatif national sur les infections transmissibles sexuellement et par le sang

Un groupe de travail composé d'experts du CCN-ITSS est formé pour traiter chacun des sujets prioritaires. D'autres contributeurs potentiels incluent des experts externes (au besoin) et des parties prenantes concernées. Le Secrétariat apporte un soutien méthodologique et technique au groupe de travail. Un examen préliminaire des données probantes est effectué afin d'identifier des revues systématiques, des lignes directrices et des études importantes publiées, ou en cours, depuis la publication de la recommandation existante ( $s$ 'il y lieu) dans le but d'aider le groupe de travail à formuler les questions de recherche (clés et contextuelles), les critères d'inclusion et d'exclusion, et le cadre d'analyse. Les résultats de cet exercice permettent également au groupe de travail de déterminer si une revue systématique est nécessaire, s'il faut utiliser ou mettre à jour une revue systématique existante ou en effectuer une nouvelle, et s'il faut adopter, adapter ou élaborer des recommandations de novo en ce qui concerne le sujet à l'étude $(17,18)$.

Le groupe de travail utilise le cadre PICO (Population, Intervention, Comparateur et Résultats/Outcomes) pour formuler la ou les questions clés, puis détermine les critères d'inclusion et d'exclusion pour l'examen des données probantes (6). Les résultats (outcomes) (bénéfiques ou nuisibles) importants pour la prise de décisions sont déterminés à partir de l'examen préliminaire des données probantes, d'une recherche ciblée de la littérature (au besoin) et des commentaires du groupe de travail et d'autres contributeurs. Le groupe de travail évalue ensuite l'importance relative de ces résultats (outcomes) pour la prise de décisions (critique, important ou non important) selon I'approche GRADE (Grading of Recommendations, Assessment, Development and Evaluation) (9). Le groupe de travail élabore également des questions contextuelles ainsi que le cadre d'analyse.

Une revue systématique est effectuée de façon indépendante par une équipe externe d'examen des données probantes. Un(e) bibliothécaire prépare la stratégie de recherche pour la revue systématique en fonction des paramètres du protocole (e.g. plans d'étude, période de temps et bases de données). Cette stratégie est ensuite examinée par un(e) deuxième bibliothécaire et le groupe de travail. Un protocole pour la revue systématique des données probantes est rédigé selon les protocoles PRISMA-P (Preferred Reporting Items for Systematic Reviews and Meta-Analysis Protocols) $(19,20)$ et comprend les questions de recherche, les critères d'inclusion et d'exclusion, le cadre d'analyse et la stratégie de recherche. La version finale du protocole est enregistrée dans la base de données PROSPERO (International Prospective Register of Systematic Reviews) (21).

Lors de la réalisation d'une revue systématique par une équipe externe, les étapes sont les suivantes : la sélection par la lecture de titres et de résumés, et de texte intégral de certains articles selon les critères d'inclusion et d'exclusion prédéfinis; l'extraction des données; l'évaluation du risque de biais; la synthèse quantitative et/ou qualitative; et l'élaboration de tableaux des données probantes en fonction de l'approche $\operatorname{GRADE}(6,9)$. La qualité ou la certitude des données probantes de chaque étude est évaluée séparément (élevée, modérée, faible ou très faible) pour chacun des résultats (outcomes) importants (9). Un article (revue systématique) est rédigé à l'aide de la liste de vérification PRISMA et le diagramme de flux PRISMA est utilisé pour présenter le processus de sélection des études (22-25). La version finale de l'article est publiée dans une revue à comité de lecture par des pairs.

Le cadre décisionnel (Evidence to Decision framework) GRADE est élaboré pour aider le groupe de travail à utiliser les données probantes de façon structurée et transparente pour formuler des recommandations $(9,26-30)$. La qualité de l'ensemble des données probantes est évaluée en fonction des résultats (outcomes) importants $(9,26-30)$.

D'autres types d'examen des données probantes, comme les revues narratives ou les revues rapides, peuvent être effectués pour certains produits d'orientation. Lorsque les données probantes issues de la littérature scientifique sont très limitées, les recommandations sont élaborées selon l'opinion d'experts en utilisant une approche systématique et transparente. 
Le groupe de travail élabore les recommandations en utilisant la terminologie de l'approche GRADE pour indiquer l'orientation et la force, le cas échéant $(9,14)$ et les présente au CCN-ITSS aux fins de discussion et de vote. L'énoncé du CCN-ITSS, élaboré par le groupe de travail, inclut le besoin des recommandations, la méthodologie utilisée, les données probantes prises en compte, les recommandations finales et un résumé des délibérations du CCN-ITSS. L'énoncé est révisé par le CCN-ITSS et publié sur le site Web canada.ca suite aux approbations de l'Agence. Les guides ITSS de l'Agence (anciennement les Lignes directrices canadiennes sur les infections transmissibles sexuellement) concernés par les recommandations sont mis à jour.

\section{Conclusion}

La méthodologie d'élaboration des recommandations du CCN-ITSS évolue afin de s'harmoniser avec les normes de pratiques exemplaires et sera améliorée au besoin. La nouvelle méthodologie, ainsi que le soutien continu et les conseils d'experts du CCN-ITSS, permet d'assurer que l'Agence fournit aux professionnels de soins de santé primaires et de santé publique des recommandations sur les ITSS qui sont fondées sur les données probantes et qui sont crédibles.

\section{Déclaration des auteurs}

S. S. - Conceptualisation, méthodologie, rédaction de l'ébauche originale, revue et révision

S. G. - Conceptualisation, rédaction de l'ébauche originale, revue et révision

M. G. R. - Conceptualisation, revue et révision

\section{Intérêts concurrents}

Aucun.

\section{Remerciements}

Les auteurs tiennent à remercier les anciens membres et les membres actuels du Comité consultatif national sur les infections transmissibles sexuellement et par le sang (CCN-ITSS) ainsi que tous ceux qui ont contribué à l'élaboration des Lignes directrices sur les ITSS. Les auteurs souhaitent aussi remercier U. Auguste et C. G. Healy d'avoir révisé la version finale de l'article.

\section{Financement}

Le travail du Comité consultatif national sur les infections transmissibles sexuellement et par le sang est financé par l'Agence de la santé publique du Canada.

\section{Références}

1. Agence de la santé publique du Canada. Nombre de cas signalés de maladies de 1924 à 2018 au Canada- maladies à déclaration obligatoire en direct. ASPC; 2020.

https://maladies.canada.ca/declaration-obligatoire/ graphiques?c=pl

2. Agence de la santé publique du Canada. Syphilis infectieuse au Canada, 2018. Relevé des maladies transmissibles au Canada 2019;45(11). Ottawa (ON) : ASPC; 2019. https://www.canada.ca/ $\mathrm{fr} /$ sante-publique/services/rapports-publications/releve-maladie s-transmissibles-canada-rmtc/numero-mensuel/2019-45/ numero-11-7-novembre-2019/article-5-rapport-syphilis-infectie use-canada-2009-2018.html

3. Agence de la santé publique du Canada. La syphilis infectieuse au Canada, 2019. Relevé des maladies transmissibles au Canada 2020;46(10). Ottawa (ON) : ASPC; 2020. https://www.canada.ca/ $\mathrm{fr} /$ sante-publique/services/rapports-publications/releve-maladie s-transmissibles-canada-rmtc/numero-mensuel/2020-46/numero10-1-octobre-2020/syphilis-infectieuse-2019.html

4. Santé et Bien-être social Canada. 1988 Directives canadiennes pour le traitement des maladies transmises sexuellement chez les nouveau-nés, les enfants, les adolescents et les adultes. Rapport hebdomadaire des Maladies au Canada. 1988;14(Suppl 2):1-20. http://publications.gc.ca/collections/collection_2016/ aspc-phac/H12-21-1-14-S2-fra.pdf

5. Agence de la santé publique du Canada. Agence de la santé publique du Canada Politique sur la gestion des organismes consultatifs externes de 2011- Sommaire. Ottawa (ON) : ASPC; 2012 (accédé 2020-05-27). https://www. canada.ca/fr/sante-publique/organisation/mandat/a-propos-agence/ organismes-consultatifs-externes/politique.htmL

6. Higgins JPT, Thomas J, Chandler J, Cumpston M, Li T, Page MJ, Welch VA, editors. Cochrane Handbook for Systematic Reviews of Interventions version 6.1 (modifié 2020-09). Cochrane, 2020. www.training.cochrane.org/handbook

7. Qaseem A, Forland F, Macbeth F, Ollenschläger G, Phillips S, van der Wees P; for the Board of Trustees of the Guidelines International Network. Guidelines International Network: toward international standards for clinical practice guidelines. Ann Intern Med 2012;156(7):525-31. DOI PubMed

8. Schünemann HJ, Al-Ansary LA, Forland F, Kersten S, Komulainen J, Kopp IB, Macbeth F, Phillips SM, Robbins C, van der Wees $P$, Qaseem A; Board of Trustees of the Guidelines International Network. Guidelines International Network: principles for disclosure of interests and management of conflicts in guidelines. Ann Intern Med 2015;163(7):548-53. DOI PubMed

9. Schünemann $H$, Brożek J, Guyatt $G$, Oxman A, editors. GRADE Handbook: handbook for grading the quality of evidence and the strength of recommendations using the GRADE approach (modifié 2013-10; accédé 2019-11-25). https://gdt.gradepro. org/app/handbook/handbook.html

10. British Association for Sexual Health and HIV. 2015 Framework for guideline development and assessment. BASHH; 2019. https://www.bashhguidelines.org/media/1229/201 5-guidelines-framework-amended-dec-2019.pdf 
11. Canadian Task Force on Preventive Health Care. Canadian Task Force on Preventive Health Care Procedure Manual. CTFPHC; 2014. https://canadiantaskforce.ca/wp-content/ uploads/2016/12/procedural-manual-en_2014_Archived.pdf

12. Desai $S$, Tunis $M$, Stirling $R$, Jensen $C$, Ismail $S$, Baclic $O$, Lerch $R$. Produits $d$ 'orientation en matière $d$ 'immunisation : différents niveaux de détail pour différents usages. Relevé des maladies transmissibles au Canada 2016;42(12):289-91. DOI

13. Healthcare Improvement Scotland. Scottish Intercollegiate Guidelines Network. SIGN 50: A guideline developer's handbook. SIGN; 2019. https://www.sign.ac.uk/assets/ sign50_2019.pdf

14. National Institute for Health and Care Excellence. Developing NICE guidelines: the manual. NICE; 2020. https://www.nice. org.uk/media/default/about/what-we-do/our-programmes/ developing-nice-guidelines-the-manual.pdf

15. United States Preventive Services Task Force. Procedure manual. USPSTF; 2015 (accédé 2019-11-25).

https://www.uspreventiveservicestaskforce.org/Page/Name/ procedure-manual

16. World Health Organization. WHO Handbook for Guideline Development, 2nd ed. Geneva (Switzerland): WHO; 2014 (accédé 2019-11-25). https://apps.who.int/iris/ handle/10665/145714

17. Schünemann HJ, Wiercioch W, Brożek J, Etxeandia-lkobaltzeta I, Mustafa RA, Manja V, Brignardello-Petersen R, Neumann I, Falavigna M, Alhazzani W, Santesso N, Zhang Y, Meerpohl JJ, Morgan RL, Rochwerg B, Darzi A, Rojas MX, Carrasco-Labra A, Adi Y, AlRayees Z, Riva J, Bollig C, Moore A, Yepes-Nuñez JJ, Cuello C, Waziry R, Akl EA. GRADE Evidence to Decision (EtD) frameworks for adoption, adaptation, and de novo development of trustworthy recommendations: GRADE-ADOLOPMENT. J Clin Epidemiol 2017;81:101-10. DOI PubMed

18. Darzi A, Harfouche $M$, Arayssi $T$, Alemadi $S$, Alnaqbi KA, Badsha $\mathrm{H}$, Al Balushi F, Elzorkany B, Halabi $\mathrm{H}$, Hamoudeh M, Hazer W, Masri B, Omair MA, Uthman I, Ziade N, Singh JA, Christiansen R, Tugwell P, Schünemann HJ, Akl EA. Adaptation of the 2015 American College of Rheumatology treatment guideline for rheumatoid arthritis for the Eastern Mediterranean Region: an exemplar of the GRADE Adolopment. Health Qual Life Outcomes 2017;15(1):183-95. DOl PubMed

19. Moher D, Shamseer L, Clarke M, Ghersi D, Liberati A, Petticrew $M$, Shekelle P, Stewart LA; PRISMA-P Group. Preferred reporting items for systematic review and meta-analysis protocols (PRISMA-P) 2015 statement. Syst Rev 2015;4(1):1-9. DOI PubMed

20. Shamseer L, Moher D, Clarke M, Ghersi D, Liberati A, Petticrew M, Shekelle P, Stewart LA; PRISMA-P Group. Preferred reporting items for systematic review and meta-analysis protocols (PRISMA-P) 2015: elaboration and explanation. BMJ 2015;349:g7647. DOI PubMed

21. National Institute for Health Research. International prospective register of systematic reviews (PROSPERO) (accédé 2020-03-27). https://www.crd.york.ac.uk/prospero/
22. Moher D, Liberati A, Tetzlaff J, Altman DG; PRISMA Group. Preferred reporting items for systematic reviews and meta-analyses: the PRISMA statement. PLoS Med 2009;6(7):e1000097. DOI PubMed

23. Liberati A, Altman DG, Tetzlaff J, Mulrow C, Gøtzsche PC, loannidis JPA, Clarke M, Devereaux PJ, Kleijnen J, Moher D. The PRISMA statement for reporting systematic reviews and meta-analyses of studies that evaluate health care interventions: explanation and elaboration. PLoS Med 2009;6(7):e1000100. DOI PubMed

24. Welch V, Petticrew M, Tugwell P, Moher D, O'Neill J, Waters E, White H; PRISMA-Equity Bellagio group. PRISMA-Equity 2012 extension: reporting guidelines for systematic reviews with a focus on health equity. PLoS Med 2012;9(10):e1001333. DOI PubMed

25. Welch V, Petticrew M, Petkovic J, Moher D, Waters E, White H, Tugwell P; the PRISMA-Equity Bellagio group. Extending the PRISMA statement to equity-focused systematic reviews (PRISMA-E 2012): explanation and elaboration. J Clin Epidemiol 2016;70:68-89. DOl PubMed

26. Andrews J, Guyatt $G$, Oxman $A D$, Alderson $P$, Dahm $P$, Falck-Ytter Y, Nasser M, Meerpohl J, Post PN, Kunz R, Brozek J, Vist G, Rind D, AkI EA, Schünemann HJ. GRADE guidelines: 14. Going from evidence to recommendations: the significance and presentation of recommendations. J Clin Epidemiol 2013;66(7):719-25. DOI PubMed

27. Andrews JC, Schünemann HJ, Oxman AD, Pottie K, Meerpohl JJ, Alonso-Coello P, Rind D, Montori VM, Brito JP, Norris S, Elbarbary M, Post P, Nasser M, Shukla V, Jaeschke R, Brozek J, Djulbegovic B, Guyatt G. GRADE guidelines: 15. Going from evidence to recommendation-determinants of a recommendation's direction and strength. J Clin Epidemiol 2013;66(7):726-35. DOI PubMed

28. Alonso-Coello $P$, Schünemann HJ, Moberg J, Brignardello-Petersen R, AkI EA, Davoli M, Treweek S, Mustafa RA, Rada G, Rosenbaum S, Morelli A, Guyatt GH, Oxman AD; GRADE Working Group. GRADE Evidence to Decision (EtD) frameworks: a systematic and transparent approach to making well informed healthcare choices. 1: Introduction. BMJ 2016;353:i2016. DOI PubMed

29. Alonso-Coello $P$, Oxman $A D$, Moberg J, Brignardello-Petersen R, Akl EA, Davoli M, Treweek S, Mustafa RA, Vandvik PO, Meerpohl J, Guyatt GH, Schünemann HJ; GRADE Working Group. GRADE Evidence to Decision (EtD) frameworks: a systematic and transparent approach to making well informed healthcare choices. 2: Clinical practice guidelines. BMJ 2016;353:i2089. DOI PubMed

30. Moberg J, Oxman AD, Rosenbaum $S$, Schünemann $H J$, Guyatt G, Flottorp S, Glenton C, Lewin S, Morelli A, Rada G, Alonso-Coello P; GRADE Working Group. The GRADE Evidence to Decision (EtD) framework for health system and public health decisions. Health Res Policy Syst 2018;16(1):45-59.

DOI PubMed 